\title{
Learning by Erasing in Dynamic Epistemic Logic
}

\author{
Nina Gierasimczuk ${ }^{\star}$ \\ Institute for Logic, Language, and Computation, University of Amsterdam \\ Institute of Philosophy, University of Warsaw \\ n.gierasimczuk@uva.nl
}

\begin{abstract}
This work provides a comparison of learning by erasing [1] and iterated epistemic update [2] as analyzed in dynamic epistemic logic (see e.g. [3]). We show that finite identification can be modelled in dynamic epistemic logic and that the elimination process of learning by erasing can be seen as iterated belief-revision modelled in dynamic doxastic logic.
\end{abstract}

Key words: identification in the limit, learning by erasing, induction, learning by elimination, co-learning, finite identifiability, dynamic epistemic logic, epistemic update, belief revision

\section{Introduction}

There have been many formal attempts to grasp the phenomenon of epistemic change. In this paper we will discuss two of them. On the one hand we have the formal learning theory (LT) framework (see e.g. [4]), with its direct implications for analysis of scientific discovery, on the other - belief-revision theory in its interrelation with dynamic epistemic logic (DEL). In learning theory, the classical framework of identification in the limit [5] was motivated mostly by the problem of language acquisition. It turned out to be very useful for modelling the process of grammar inference, and found numerous applications in the area of syntax. Initially the idea of identification was unappreciated in semantic considerations, but eventually also this direction has started to be developed resulting in applications to the acquisition of semantics of natural language [6-8] as well as in modelling the process of scientific inquiry [9]. The serious step towards involving more semantics was coupled with the design of model-theoretic learning [10] and its application to belief-revision theory [11].

Other, very prominent directions that explicitly involve notions of knowledge and belief have been developed in the area of epistemology. First, a precise language to discuss epistemic states of agents has been established in [12]. After that the need of formalizing dynamics of knowledge emerged. The belief-revision AGM framework [13] constitutes an attempt to talk about the dynamics of epistemic states. Belief-revision policies thus explained have been successfully

* The author is a receiver of the Foundation for Polish Science Award for Young Researchers (START Programme 2008). 
modelled in dynamic epistemic logic (see [3]) and in the above-mentioned modeltheoretic learning [11].

In the present paper we show how those two important traditions, LT and DEL, can be merged. We explain this connection by joining iterated epistemic update as modelled in DEL with a special case of learning in the limit - learning by erasing $[14,1]$.

We will proceed according to the following plan. First we explain the ideas of dynamic epistemic logic (DEL) from a strictly semantic point of view. We will also mention an important modification of DEL, namely dynamic doxastic logic (DDL). As we will see the part 'dynamic' in those names refers to the fact that those logics include operators which modify models. With respect to those modifications we discuss the notions of epistemic and doxastic update. In particular, we focus on public announcement as a special case. Then we leave this logical subject and move to briefly recall the basics of formal learning theory in its set-theoretical version. After that the definition of learning by erasing is provided. The last two parts present a way to model finite identification in DEL and learning by erasing in DDL.

\section{Dynamic Epistemic Logic. Semantic Perspective}

In general, dynamic epistemic logic has been introduced to formalize knowledge change. In this section basic notions of DEL will be provided. The definitions are based on [15]. Let us take Atom to be a set of atomic propositions and $A-$ a set of agents.

\section{Definition 1 (Epistemic Model).}

Epistemic model $M$ is a triple $\left\langle W,\left\{\sim_{i}\right\}_{i \in A}, V\right\rangle$, where $W$ is a set of possible worlds, for each $i \in A, \sim_{i} \subseteq W \times W$ is an indistinguishability relation and $V:$ Atom $\rightarrow \wp(W)$ is a valuation.

Intuitively speaking, $M$ formalizes the epistemic situation of all agents from $A$. The indistinguishability relation models their uncertainty about which of the possible worlds is the actual one.

Definition 2 (Event Model). An event model $E$ is a triple $\left\langle S,\left\{\rightarrow_{i}\right\}_{i \in A}\right.$, pre $\rangle$, where $S$ is a set of worlds, for each $i \in A, \rightarrow i \subseteq S \times S$, and pre $: S \rightarrow$ Atom is a pre-condition function which indicates what pre-condition a world has to satisfy to enable the event to take place.

Event model describes the epistemic content of the event. Relation $\rightarrow_{i}$ directly corresponds to the indistinguishability relation $\sim_{i}$ of epistemic model.

\section{Definition 3 (Product Update).}

Let $M=\left\langle W,\left\{\sim_{i}\right\}_{i \in A}, V\right\rangle$ and $E=\left\langle S,\left\{\rightarrow_{i}\right\}_{i \in A}\right.$, pre $\rangle$. The product update $M \otimes E$ is the epistemic model $M^{\prime}=\left\langle W^{\prime},\left\{\sim_{i}^{\prime}\right\}_{i \in A}, V^{\prime}\right\rangle$ such that:

$$
\text { - } W^{\prime}=\{(w, s) \mid w \in W, s \in S \text { and } M, w \models \operatorname{pre}(s)\},
$$


- $(w, s) \sim_{i}\left(w^{\prime}, s^{\prime}\right)$ iff $w \sim_{i} w^{\prime}$ and $s \rightarrow_{i} s^{\prime}$,

$-V^{\prime}((w, s))=V(w)$.

Definition 4 (Public Announcement [16]). The public announcement of a formula $\varphi$ is the event model $E_{\varphi}=\left\langle S,\left\{\rightarrow_{i}\right\}_{i \in A}\right.$, pre $\rangle$, such that $S=\{e\}$ and for each $i \in A, e \rightarrow_{i} e$ and $\operatorname{pre}(e)=\varphi$.

The major result of updating an epistemic model $M$ with public announcement of $\varphi$ is a submodel of $M$ containing only the states that satisfy $\varphi$.

Example 1. Let us take the set of agents $A=\{a$ (Anne), $b$ (Bob), $c$ (Carl) $\}$ and the deck of cards consisting of: 1, 2, 3. Each person gets one card. We can represent the situation after dealing as a triple $x y z$, where $x, y, z$ are cards and the first position in the triple assigns the value to $a$ (Anne), second to $b$ (Bob), etc. For instance, 231 means that Anne has 2, Bob has 3 and Carl has 1. All possible situations after a deal are: 123, 132, 213, 231, 312, 321. We assume that all the players are witnessing the fact of dealing but they do not know the distribution of the cards. The epistemic model $M$ of this situation is illustrated in the figure.

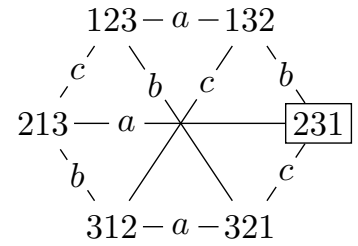

Let us then assume that as a result the actual world is 231. Obviously each player's knowledge does not allow certainty about which is the actual world. In the model the uncertainty of the agent $x$ about the worlds $w$ and $w^{\prime}$ is symbolized by the following: $w \sim_{x} w^{\prime}$ (in the Figure this relation is depicted by two states being joined by a line labeled with $x$ ).

Let us now assume that Anne shows her card to all the players publicly, i.e., all the players see her card and all of them know that all of them see it. This event is modelled by $E=\left(S,\left\{\rightarrow_{i}\right\}_{i \in A}\right.$, pre $)$, where $S=\{s\}$, for each $x \in A$, $s \rightarrow_{x} s$ and $\operatorname{pre}(s)=2_{-}$('Anne has 2 ').

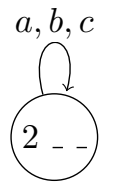

The public announcement of 'Anne has 2' results in the epistemic situation, which can be presented as $M^{\prime}=M \otimes E$ (depicted below).

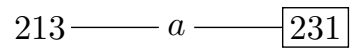


Event $E$ is an example of a public announcement, in this case: 'Anne has 2 '. In dynamic epistemic logic the public announcement of $\varphi$ is represented by ' $\varphi$ ' and corresponds to the elimination of all those possible worlds that do not satisfy $\varphi$. In other words, public announcement works as relativization of the model to those worlds that satisfy the content of the announcement.

\section{Dynamic Doxastic Logic}

The objective of dynamic doxastic logic (DDL) is to formalize the notion of belief change. This is usually done by introducing preference relations over the possible worlds. Each agent has his own preference relation. Belief of agent $a$ is determined by the set of his most preferred states.

Definition 5 (Epistemic Plausibility Model). Let Atom be a set of atomic propositions and $A-$ a set of agents. Epistemic plausibility model $E$ is a quadruple: $\left\langle W,\left\{\sim_{i}\right\}_{i \in A},\left\{\leq_{i}\right\}_{i \in A}, V\right\rangle$, where $W$ is a set of possible worlds, for each $i \in A, \sim_{i} \subseteq W \times W$ is an indistinguishability relation, $\leq_{i} \subseteq W \times W$ is a preference relation and $V:$ Atom $\rightarrow \wp(W)$ is a valuation.

Definition 6 (Plausibility Event Model). An event model $E$ is a quadruple: $\left\langle S,\left\{\rightarrow_{i}\right\}_{i \in A},\left\{\preceq_{i}\right\}_{i \in A}\right.$, pre $\rangle$, where $S$ is a set of worlds, for each $i \in A, \rightarrow_{i} \subseteq$ $S \times S, \preceq_{i} \subseteq S \times S$ and pre $: S \rightarrow$ Atom is a pre-condition function.

For completeness' sake we add the definition of priority update.

Definition 7 (Priority Update). The priority update works analogously to the epistemic update. The additional condition is for the $\leq_{i}$ relation:

- for $w \in W$ and $s \in S,(w, s) \leq_{i}{ }_{i}\left(w^{\prime}, s^{\prime}\right)$ iff $s \prec_{i} s^{\prime}$, or $s \simeq_{i} s^{\prime}$ and $w \leq_{i} w^{\prime}$, where $s \simeq_{i} s^{\prime}$ iff $s \preceq_{i} s^{\prime}$ and $s^{\prime} \preceq_{i} s$.

\section{Learning Theory}

\subsection{Identification in the Limit}

Learning theory is concerned with the process of inductive inference [5]. We can think of it as of a game between Scientist and Nature. In the beginning we have a class of possible worlds together with a class of hypotheses (possible descriptions of worlds). Different hypotheses may describe the same world. We assume that both Scientist and Nature know what all the possibilities are, i.e., they both have access to the initial classes. Nature chooses one of those possible worlds to be the actual one. Scientist has to guess which it is. Scientist receives information about the world in an inductive manner. The stream of data is infinite and contains only and all the elements from the chosen reality. Each time Scientist receives a piece of information he answers with one of the hypotheses from the initial class. We say that Scientist identifies Nature's choice in the limit if after some finite number of guesses his answers stabilize on a correct hypothesis. Moreover, 
to discuss more general identifiability, we require that the same is true for all the possible worlds from the initial class, i.e., regardless of which element from the class is chosen by Nature to be true, Scientist can identify it in the limit on the basis of data about the actual world.

To formalize this simple setting we need to make the notion of stream of data clear. In learning theory such streams are often called 'environments' ${ }^{1}$.

Let us consider $E$ - the set of all computably enumerable sets. Let $C \subseteq E$ be some class of c.e. sets. For each $S$ in $C$ we consider Turing machines $h_{n}$ which generate $S$ and in such a case we say that $n$ is an index of $S$. The Turing machines will function as the conjectures that Scientist makes. It is well-known that each $S$ has infinitely many indices. Let us take $I_{S}$ to be the set of all indices of the set $S$, i.e, $I_{S}=\left\{n \mid h_{n}\right.$ generates $\left.S\right\}$.

Definition 8 (Environment). By environment of $S$, $\varepsilon$, we mean any infinite sequence of elements from $S$ such that:

1. $\varepsilon$ enumerates all the elements from $S$;

2. $\varepsilon$ enumerates only the elements from $S$;

3. $\varepsilon$ allows repetitions.

Definition 9 (Notation). We will use the following notation:

- $\varepsilon_{n}$ is the $n$-th element of $\varepsilon$;

- $\varepsilon \mid n$ is a sequence $\left(\varepsilon_{0}, \varepsilon_{1}, \ldots, \varepsilon_{n-1}\right)$;

- $S E Q$ denotes the set of all finite initial segments of all environments;

- $\operatorname{set}(\varepsilon)$ is a set of elements that occur in $\varepsilon$;

- $h_{n}$ will refer to a hypothesis, i.e., a finite description of a set, a Turing machine generating $S$;

- L is a learning function - a map from finite data sequences to indices of hypotheses, $L: S E Q \rightarrow I_{H_{C}}$.

The structure of the identifiability in the limit can be formulated by the following chain of definitions:

Definition 10 (Identification in the limit, LIM).

We say that a learning function $L$ :

1. identifies $S \in C$ in the limit on $\varepsilon$ iff there is a number $k$, such that for co-finitely many $m, L(\varepsilon \mid m)=k$ and $k \in I_{S}$;

2. identifies $S \in C$ in the limit iff it identifies $S$ in the limit on every $\varepsilon$ for $S$;

3. identifies $C$ in the limit iff it identifies in the limit every $S \in C$.

The notion of identifiability can be strengthened in various ways. One radical case is to introduce a finiteness condition for identification.

Definition 11 (Finite identification, FIN).

We say that a learning function $L$ :

1. finitely identifies $S \in C$ on $\varepsilon$ iff, when successively fed $\varepsilon$, at some point $L$ outputs a single $k$, such that $k \in I_{S}$, and stops;

2. finitely identifies $S \in C$ iff it finitely identifies $S$ on every $\varepsilon$ for $S$;

3. finitely identifies $C$ iff it finitely identifies every $S \in C$.

\footnotetext{
${ }^{1}$ We are concerned here only with sequences of positive information (texts).
} 


\subsection{Learning by Erasing}

Learning by erasing $[1,17]$ is an epistemologically intuitive modification of identification in the limit. Very often the cognitive process of converging to a correct conclusion consists of eliminating those possibilities that are falsified during the inductive inquiry. Accordingly, in the formal model the outputs of the learning function are negative, i.e., the function each time eliminates a hypothesis, instead of explicitly guessing one that is supposed to be correct. A special case of learning by erasing is co-learning [14]. The set $S \in C$ is co-learnable iff there is a function which stabilizes by eliminating all indices from $I_{H_{C}}$ except just one from $I_{S}$. The difference between this approach and the usual identification is in the interpretation of the positive guess of the learning function. In learning by erasing there is always some ordering of the initial hypothesis space. This allows to interpret the actual positive guess of the learning-by-erasing function to be the least hypothesis (in a given ordering) not yet eliminated.

Let us give now the two definitions that explain the notion of learning by erasing.

Definition 12 (Function Stabilization). In learning by erasing we say that a function stabilizes to number $k$ on environment $\varepsilon$ if and only if for co-finitely many $n \in \mathbb{N}$ :

$$
k=\min \{\mathbb{N}-\{L(\varepsilon \mid 0), \ldots, L(\varepsilon \mid n)\}\} .
$$

Definition 13 (Learning by Erasing, E-learning).

We say that a learning function, $L$ :

1. learns $S \in C$ by erasing on $\varepsilon$ iff $L$ stabilizes to $k$ on $\varepsilon$ and $k \in I_{S}$;

2. learns $S \in C$ by erasing iff it learns by erasing $S$ from every $\varepsilon$ for $S$;

3. learns $C$ by erasing iff it learns by erasing every $S \in C$.

A variety of additional conditions for learning can be defined. Let us mention the following conditions on e-learning function $L[1]$.

1. $L$ erases all but one, correct hypothesis (co-learning, e-ALL);

2. $L$ erases only hypotheses that are incorrect (e-SUB);

3. $L$ erases exactly all hypotheses that are incorrect (e-EQ);

4. $L$ erases all hypotheses that are incorrect but may also erase some that are correct (e-SUPER);

Let us cite two theorems [1] that establish the relationships between various types of learning: e-learning, finite identifiability and identifiability in the limit.

Theorem 1. FIN $\subset e-E Q \subset e-S U B \subset L I M$

Theorem 2. e-ALL, e-SUPER $=L I M$ 


\section{Finite Identification in DEL}

The word 'learning' is used in epistemology to cover a variety of epistemic processes. One of them is the epistemic update in the form of one-step learning that $\varphi$, followed by a direct modification of the set of beliefs, as we have seen in sections 2 and 3 . In the learning-theoretic setting the incoming information is of a different nature than the actual thing being learned. This feature has an important consequence for modelling learning in DEL. We are forced to provide two-sorted models, with one sort for pieces of incoming information and another for the hypotheses. To establish a bridge between those two different ontologies we treat a hypothesis as the set of events that it predicts, e.g., if we take a hypothesis $h$ to be 'There are all natural numbers except 3' it predicts that the environment will enumerate all the natural numbers except 3.

The possible worlds in our epistemic model are identified with hypotheses. Unlike in the classical DEL approach, the event models are announcements of data corresponding to elements of the sets being learned, and not hypotheses themselves.

A further difference is in the number of agents. In sections 2 and 3 we provided definitions for multi-agent epistemic cases. Although science as well as learning seem to be at least a two-player game, in the present paper we are concerned only with the role of Scientist (Learner). By implication, we assume Nature (Teacher) to be an objective machine that makes an arbitrary choice and gives out random data, she does not have any particular strategy, is neither helping the learner, nor obstructing his attempts to identify a correct hypothesis. We recognize the possibility and potential of analyzing two or more agents in the contexts of inductive inference. However, for the sake of simplicity our DEL and DDL models are going to account only for one agent.

Let us again fix $C$ to be a class of sets, and for each $S_{n} \in C$ we consider $h_{n}$ to be a hypothesis that describes $S_{n}$. In learning by erasing we can take the initial epistemic model to represent the background knowledge of Scientist together with his uncertainty about which world is the actual one. Let us take the initial epistemic frame to be

$$
M=\left\langle H_{C}, \sim\right\rangle,
$$

where $H_{C}$ is a possibly infinite ${ }^{2}$ set of worlds (hypotheses that are considered possible) and $\sim \subseteq H_{C} \times H_{C}$ is an uncertainty relation for Scientist. Since we assume that the initial hypothesis space is arbitrary, we also do not require any particular preference of the scientist over $H_{C}$. Hence, we take the relation $\sim$ to be a universal, equivalence binary relation over $H_{C}$. The initial epistemic state of the Scientist is depicted in Figure 1. This model corresponds to the starting point of the scientific discovery process. Each world represents a hypothesis from the initial set determined by the background knowledge. In the beginning Scientist

\footnotetext{
${ }^{2}$ We can effectively deal with the epistemic update and identification in infinite domains by using special enumeration strategies (for explanation and examples see $[18])$.
} 
considers all of them possible. The model also reflects the fact that Scientist is given the class of hypotheses $H_{C}$. In other words he knows what the alternatives are.

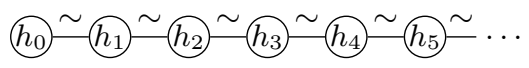

Fig. 1. Initial epistemic model

Next, Nature decides on some state of the world by choosing one possibility from $C$. Let us assume that as a result $h_{3}$ correctly describes the chosen world. Then, she decides on some particular environment $\varepsilon$, of the elements from the world. We picture this enumeration in Figure 2 below.

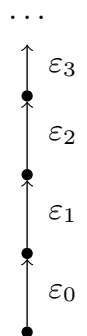

Fig. 2. Environment $\varepsilon$ consistent with $h_{3}$

The sequence $\varepsilon$ is successively given to Scientist. Let us focus now on the first step of the procedure. We have the uncertainty range of Scientist, it runs through the whole set of hypotheses $H_{C}$. A piece of data $\varepsilon_{0}$ is given to Scientist. This fact can be represented by the event model $E_{0}=\langle\{e\}, \rightarrow$,pre $\rangle$, where $e \rightarrow e$ and $\operatorname{pre}(e)=\varepsilon_{0}$ (see Figure 3 ).

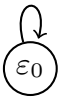

Fig. 3. Event model $E_{0}$ of the announcement of $\varepsilon_{0}$

Scientist, when confronted with the announcement of $\varepsilon_{0}$ updates his epistemic state accordingly. We will represent the process formally by the product update $M \otimes E_{0}$. The result of the product update is again an epistemic model $M^{\prime}=\left\langle H_{C}{ }^{\prime}, \sim^{\prime}\right\rangle$, where: 
1. $\left.H_{C}{ }^{\prime}=\left\{\left(h_{n}, e\right) \mid h_{n} \in H_{C} \& \operatorname{pre}(e) \in S_{n}\right)\right\}$;

2. $\sim^{\prime}=\sim \mid H_{C}{ }^{\prime}$.

We use here event models similar in spirit to those of public announcement [16]. They consist in only one state with a pre-condition determined by the piece of data that is given. In Figure 4 Scientist's confrontation with $\varepsilon_{0}$ is depicted.

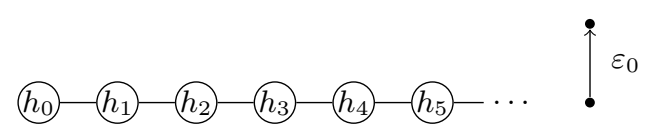

Fig. 4. Confrontation with data

Scientist tests each hypothesis with $\varepsilon_{0}$. If a hypothesis is consistent with it, it remains as a possibility, if it is not consistent, it is eliminated (see figure 5). Let us assume that $\varepsilon_{0}$ is not consistent with $h_{2}$.

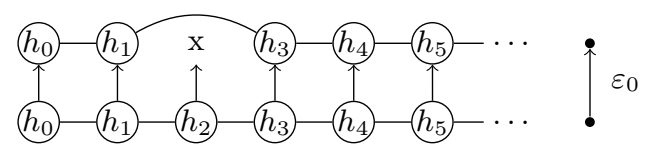

Fig. 5. Epistemic update

This epistemic update can be iterated infinitely many times along $\varepsilon$ resulting in an infinite sequence of models which according to the lines of DEL can be called $\varepsilon$-generated epistemic model (see e.g. [15]).

Definition 14 (Generated Epistemic Model).

The generated epistemic model $(M)^{\varepsilon}$, with $\varepsilon=\varepsilon_{0}, \varepsilon_{1}, \varepsilon_{2}, \ldots$, is the result of epistemic update $M \otimes E_{0} \otimes E_{1} \otimes E_{2} \otimes \ldots$, where for each $n$, the event $E_{n}$ corresponds to the announcement of $\varepsilon_{n}$.

Let us now see a simple example of finite identification of a single hypothesis.

Example 2. Let us take $H_{C}=\left\{h_{0}, h_{1}, h_{2}\right\}$, such that $h_{n}=\{0, \ldots, n\}$. Nature makes her choice regarding what the world is like. We assume that as a result $h_{2}$ holds. Then, Nature chooses an enumeration $\varepsilon=0,1,0,2,1, \ldots$. After the first piece of data, 0 , the uncertainty range of Scientist includes the whole $H_{C}$. After the second, 1 , Scientist eliminates $h_{0}$ since it does not contain the event 1 and now he hesitates between $h_{1}$ and $h_{2}$. The third piece, 0 , does not change anything, however the next one, 2 , eliminates $h_{1}$. Uncertainty is eliminated as well. He knows that the only hypothesis that can be true is $h_{2}$. Therefore, we can say that he learned it conclusively, with certainty.

The above discussion suggests the following thesis. 
Thesis 1 Finite identifiability can be modelled within the DEL framework, using:

- epistemic states for hypotheses;

- infinite sequences of announcements for environments;

- epistemic update for the progress in eliminating uncertainty over hypothesis space.

Scientist succeeds in finite identification of $S$ from $\varepsilon$ if and only if there is a finite initial segment of $\varepsilon, \varepsilon \mid n$, such that the domain of the $\varepsilon \mid n$-generated model contains only one hypothesis $h_{k}$ and $k \in I_{S}$. In other words, there is a finite step of the iterated epistemic update along $\varepsilon$, that eliminates Scientist's uncertainty.

\section{Learning by Erasing in DDL}

From Scientist's point of view the process of learning has a few components that are very important in logical modelling. The first is of course the current conjecture - a hypothesis that is considered appropriate in a given step of the procedure. The second is the set of those hypotheses that were used in the past and have already been discarded. The third part is the set of hypotheses that are still considered to be possible, but for some reasons less probable than the chosen one.

Let us consider the following example of a learning scenario, in which the uncertainty is never eliminated.

Example 3. As you probably observed, in the Example 2 Scientist was very lucky. Let us assume for a moment that nature had chosen $h_{1}$, and had fixed the enumeration $\varepsilon=0,1,0,1,1,1,1, \ldots$ In this case Scientist's uncertainty can never be eliminated. ${ }^{3}$

This example indicates that the central element of the identification in the limit model is the unavoidable presence of uncertainty. The limiting framework allows however the introduction of some kind of operational knowledge, which is uncertainty-proof.

To model the algorithmic nature of the learning process that includes actual guess and other not-yet-eliminated possibilities, we enrich the epistemic model with some preference relation $\leq: H_{C} \times H_{C}$. The relation $\leq$ represents some preference over the set of hypotheses, e.g., if Scientist is an occamist, the preference would be defined according to the simplicity of hypotheses. In the initial epistemic state the uncertainty of the scientist again ranges over all of $H_{C}$. This time however the class is ordered and Scientist current belief is the most preferred hypothesis. Therefore, we consider the initial epistemic state of Scientist to be:

$$
M=\left\langle H_{C}, \sim, \leq\right\rangle .
$$

\footnotetext{
${ }^{3}$ As we are interested here in learning by erasing, we assume a suitable underlying ordering of hypothesis space. In this case it is: $h_{0}, h_{1}, h_{2}$. However, note that this type of identification is not order-independent. If the initial ordering was: $h_{0}, h_{2}, h_{1}$, then Scientist would not stabilize on the correct hypothesis.
} 
The procedure of erasing hypotheses that are inconsistent with successively incoming data is the same as in the previous section. This time however let us introduce the current-guess state which is interpreted as the actual guess of the Scientist. It is always the one that is most preferred - the smallest one according to $\leq$. In doxastic logic a set of most preferred hypotheses is almost invariably interpreted as the one that the agent believes in. Let us go back to Example 2, where Nature chose a world consistent with $h_{1}$. After seeing 1 and eliminating $h_{0}$, Scientist's attention focuses on $h_{1}$, then $h_{1}$ is his current belief. It is the most preferred hypothesis, and as such it can be reiterated as long as it is consistent with $\varepsilon$. In this particular case, since Nature chose a world consistent with $h_{1}$, it will never be contradicted, so Scientist will always be uncertain between $h_{1}$ and $h_{2}$. However, his preference directs him to believe in the correct hypothesis, without him being aware of the correctness. Therefore, we claim the following.

Thesis 2 Learning by erasing can be modelled within the DDL framework, using:

- epistemic states for hypotheses;

- infinite sequences of announcements for environments;

- epistemic update for the progress in eliminating uncertainty over the hypothesis space;

- preference relation for the underlying hypothesis space;

- in each step of the procedure, the most preferred hypothesis for the actual positive guess of the learning function.

Scientist learns $S$ by erasing from $\varepsilon$ if and only if there is $n$ such that for every $m>n$, the most preferred state of the domain of the $\varepsilon \mid m$-generated epistemic model is $h_{k}$, and $k \in I_{S}$.

\section{Conclusions and Further Work}

In this paper we argued that the process of inductive inference can be modelled in dynamic epistemic logic and dynamic doxastic logic. To support our claim we provided a translation of the components of learning into a two-sorted semantics for DEL and DDL. In particular, we see DEL as an appropriate framework to analyze the notion of finite identifiability. Learning by erasing, a special case of identifiability in the limit, is based on the existence of an underlying ordering of hypothesis space. Therefore, in logical modelling it requires adding to the epistemic model a preference relation over possible worlds. This indicates that it should be formalized in DDL, where the preference relation is a standard element of any model.

The above-presented conceptual work has many implications and possible continuations. After establishing a correspondence on the semantic level, it is possible to formulate axioms of epistemic logic for inductive inference. We find this project promising and potentially fruitful for both DEL and LT. Moreover, modal analysis of the process of learning can be continued in the following directions: 
- formulating LT theorems as validities in epistemic and temporal logic;

- analyzing the inductive inference process in game-theoretical terms, and discussing strategies for learning and teaching;

- studying the notion of non-introspective operational knowledge and uncertainty that are involved in the process of inductive inference;

- comparing formal learning theory and belief-revision theory in a systematic way.

\section{References}

1. Lange, S., Wiehagen, R., Zeugmann, T.: Learning by erasing. In: Proc. 7th Int. Workshop on Algorithmic Learning Theory. Volume 1160 of LNAI., SpringerVerlag (1996) 228-241

2. Baltag, A., Moss, L.: Logics for epistemic programs. Synthese 139(2) (2004) $165-224$

3. van Ditmarsch, H., van der Hoek, W., Kooi, B.: Dynamic Epistemic Logic. Springer (2007)

4. Jain, S., Osherson, D., Royer, J.S., Sharma, A.: Systems that Learn. MIT Press (1999)

5. Gold, E.: Language identification in the limit. Information and Control 10 (1967) 447-474

6. Tiede, H.J.: Identifiability in the limit of context-free generalized quantifiers. Journal of Language and Computation 1 (1999) 93-102

7. Costa Florêntio, C.: Learning generalized quantifiers. In: Proc. 7th ESSLLI Student Session. (2002)

8. Gierasimczuk, N.: The problem of learning the semantics of quantifiers. In: Logic, Language, and Computation, 6th TbiLLC 2005. Volume 4363 of LNAI., Springer (2007) 117-126

9. Kelly, K.: The Logic of Reliable Inquiry. Oxford University Press (1996)

10. Osherson, D., de Jongh, D., Martin, E., Weinstein, S.: Formal learning theory. In van Benthem, J., Ter Meulen, A., eds.: Handbook of Logic and Language. MIT Press (1997) $737-775$

11. Martin, E., Osherson, D.: Elements of Scientific Inquiry. MIT Press (1998)

12. Hintikka, J.: Knowledge and Belief. An Introduction to the Logic of the Two Notions. Cornell University Press (1962)

13. Alchourrón, C.E., Gärdenfors, P., Makinson, D.: On the logic of theory change: Partial meet contraction and revision functions. The Journal of Symbolic Logic 50(2) (1985) 510-530

14. Freivalds, R., Zeugmann, T.: Co-learning of recursive languages from positive data. In: Perspectives of System Informatics, Proc. 2nd Andrei Ershov Memorial Conference. Volume 1181 of LNCS., Springer (1996) 122-133

15. van Benthem, J., Gerbrandy, J., Pacuit, E.: Merging frameworks for interaction: DEL and ETL. In: Proc. TARK'07. (2007) 72-81

16. Batlag, A., Moss, L.S., Solecki, S.: The logic of public announcements and common knowledge and private suspicions. In: Proc. TARK'98. (1998) 43-56

17. Freivalds, R., Karpinski, M., Smith, C., Wiehagen, R.: Learning by the process of elimination. Information and Computation 176(1) (2002) 37-50

18. Gierasimczuk, N.: Identification through inductive verification. In: 7th TbiLLC 2007. Volume 5422 of LNAI., Springer (2009) 193-205 